\title{
INTEGRATING EXPERIENTIAL LEARNING: USING AN EHR SYSTEM TO TEACH CDSS IN HEALTH INFORMATICS
}

\author{
Kalyani Ankem, Northern KentuckyUniversity, ankemk1@nku.edu \\ Vishal Uppala, North Dakota State University, vishal.uppala@ndsu.edu \\ Adam Durst, MercyHealth, amdurst@mercy.com
}

\begin{abstract}
Experiential learning is critical in preparing health informatics students for the workplace. Although health informatics has evolved to developing information systems that optimize patient care and patient engagement, an understanding of the EHR system is essential for students. A hands-on component in performing tasks using the CDSS in the EHR system was designed to assess the experiential learning gained by students. Pre- and post-test questions to assess students' understanding of the CDSS were administered. Additionally, at post-test, students responded to questions on student engagement, student learning, and student interest in extended learning. Students' understanding of the CDSS significantly increased at post-test $(p<.01)$. Open-ended responses confirm their improved understanding of CDSS concepts and functionality. Students found the hands-on CDSS component to be engaging. Also, students believed the hands-on component contributed to learning as well as an interest in learning more about the CDSS.
\end{abstract}

Keywords: Experiential Learning, Health Informatics, Electronic Health Record (EHR) System, Clinical Decision Support System (CDSS)

\section{INTRODUCTION}

Electronic health record (EHR) skills are fundamental in the preparation of health informatics students. Although health informatics has progressed to leveraging clinical data collected to inform decision-making through analytics, EHR skills continue to be essential in building a more advanced skill set. EHR skills benefit students in not only healthcare majors such as nursing and allied health but also information technology majors such as information systems and computer science.

The EHR is a digital record of a patient's medical and treatment histories. Clinical data collected in the EHR includes "medical history, diagnoses, medications, treatment plans, immunization dates, allergies, radiology images, and laboratory and test results" (HealthIT.gov, 2018b). While the EHR enables clinical data collection, it is also designed to go beyond this function by integrating critical clinical decision support tools that providers can use to improve patient care (HealthIT.gov, 2018b). The EHR, in effect, streamlines the provider's workflow (HealthIT.gov, 2018b; HIMSS, 2017). Also, the digital format of the EHR, which contains health information created and managed by authorized providers, revolutionizes healthcare as it facilitates the capability of sharing health information among providers across healthcare organizations. As such, the centrality of this record in patient care renders knowledge of the EHR and integrated technologies essential for the health information technology (IT) workforce as well as healthcare professionals.

To educate and develop EHR skills among students preparing to work in healthcare, an EHR system was implemented in our health informatics program for teaching purposes. A detailed description of the implementation can be found in Ankem, Uppala, and Dhawan (2017). This research builds upon the earlier implementation and employs the experimental method to assess the effectiveness of using the EHR system in a health informatics course. A clinical decision support system (CDSS) hands-on component was designed and introduced to students.

A CDSS often is a health IT component of the EHR system. The CDSS "provides clinicians, staff, patients or other individuals with knowledge and person-specific information, intelligently filtered or presented at appropriate times, to enhance health and health care" (HealthIT.gov, 2018a). The CDSS is a sophisticated technology which combines 


\section{Issues in Information Systems}

Volume 20, Issue 3, pp. 194-207, 2019

biomedical knowledge base with a patient's clinical information and uses reasoning or inference to generate and present information to users in the clinical workflow. It is a variation of the decision support system (DSS) designed to support business management.

The CDSS offers several tools to improve the clinical decision-making process (AHRQ, 2018). CDSS tools include medication ordering, knowledge support, medical calculators, trending/patient tracking, order sets/protocols for particular conditions, reminders for preventative care, differential diagnosis, radiology support, laboratory support, potentially dangerous situation alerts, and public health alerts. The CDSS hands-on component in the present study included use of the medication ordering support and knowledge support tools of the CDSS. These CDSS tools assist in avoiding errors and adverse events, thereby, increasing the quality of care and improving patient outcomes. Students in nursing may seek responsibilities that include prescribing medications which may require them to use the medication ordering support and knowledge support tools of the CDSS. Other students in healthcare majors, such as those in allied health, can also benefit from the hands-on experience. These students will be in positions where they will observe alerts and reminders on a regular basis and may work with the radiology or laboratory support tools of the CDSS. Hence, understanding how the CDSS operates is beneficial to them. Students in information technology majors such as information systems and computer science can experience a functional CDSS and perhaps contribute to the design and development of these information technologies in the future.

Upon students' completion of the CDSS hands-on component using the EHR system, the t-test was applied to find a difference in students' understanding of CDSS concepts and functionality between pre- and post-tests. Jewer and Evermann's (2015) instrument was adapted to gather data from students. Students' open-ended responses to demonstrate understanding of CDSS concepts and functionality were assessed qualitatively to find if understanding increased from pre- to post-test. Means and standard deviations were used to examine student engagement, student learning, and student interest in learning more as a result of students gaining hands-on experience with the CDSS.

\section{EHR INTEGRATION IN TEACHING}

The integration of EHR systems in teaching, however, has been challenging in the education of health IT professionals as well as healthcare professionals. Healthcare professionals, especially those who spend their time in patient care, are rarely prepared to use the EHR optimally to realize the efficiencies and improvements in patient outcomes. Undergraduate medical students, in particular, are rarely exposed to EHR training in their education (Borycki et al., 2009; Borycki, Joe, Armstrong, Bellwood, \& Campbell, 2011; Otto \& Kushniruk, 2009), which removes them from the situational learning of "real world" clinical practice. Biagioli and colleagues found the EHR experience to be lacking among clerkship students (Biagioli et al., 2017). According to Biagioli et al., while the clerkship students performed well with communication tasks, their data management competencies were deficient. Other authors have called for hands-on experience in medical education to not only balance EHR documentation with patient engagement but also to learn order entry in the EHR and optimally utilize clinical decision support tools. According to Welcher and colleagues, "Medical students need hands-on experience documenting clinical encounters as well as entering orders to prepare for residency and become competent physicians" (Welcher, Hersh, Takesue, Stagg Elliott, \& Hawkins, 2018, p. 48). The researchers state that medical students are underprepared for practice when they lack EHR access. Electronic health records (EHRs) bring transformational change in healthcare and have an undeniable impact on patient care. Nevertheless, teaching optimal use of EHRs in medical education receives less than desired attention and remains unintegrated in spite of numerous calls for such integration to become routine (Kushniruk et al., 2012; Milano, Hardman, Plesiu, Rdesinski, \& Biagioli, 2014).

Likewise, the integration of health IT remains a challenge in nursing and health informatics education. Herbert and Connors (2016) found that $49 \%$ of faculty in undergraduate nursing programs surveyed had an awareness of EHRs for academic use. A small percentage, $9 \%$, had decided to use them on a small scale, and only $9 \%$ fully utilized them. It is a challenge for nursing programs to integrate health IT in the curriculum due to technical, financial and knowledge limitations (Kushniruk, Kuo, Parapini, \& Borycki, 2014). In other disciplines, a similar survey of the extent of EHR integration in teaching across multiple programs has not yet been reported.

A few descriptions of EHR integration within a program in a university do exist in the literature. Some articles discuss integration in medical education, mostly focusing on EHR skills deemed important in training medical students 


\section{Issues in Information Systems \\ Volume 20, Issue 3, pp. 194-207, 2019}

(Milano et al., 2014). The School of Health Information Science at the University of Victoria describes the use of EHRs in a broader context in teaching nursing, medical, and health informatics students (Borycki et al., 2009; Borycki et al., 2011; Borycki, Griffith, Reid, Kushniruk, \& Kuo, 2013; Kushniruk et al., 2012; Kushniruk et al., 2014). Nursing and undergraduate medical students learn about the EHR with an emphasis on healthcare practice whereas health informatics students learn about the EHR with an emphasis on information technology. More specifically, teaching health informatics students focuses on usability, databases, and programming to develop modules that can interface with the EHR. Using a simulated EHR has been a common practice in these universities in delivering this EHR experience to students, mainly due to HIPAA policies and regulations.

EHR use in health informatics programs within computer science or information systems departments is rarely reported. Often, students gain the hands-on experience with EHR systems through practicums or internships in healthcare settings (Wilson \& Tulu, 2010). In one of the few articles that address such integration, Zhang et al. reported that the health informatics program in the School of Computing and Software Engineering at Southern Polytechnic State University incorporates several commercial as well as open-source EHRs in teaching (Zhang, Reichgelt, Rutherfoord, \& Wang, 2014). Zhang et al., however, do not discuss the details of this integration.

Although the field of health informatics has moved to improving information systems for better patient care and patient engagement and for leveraging robust data analytics for better patient outcomes, knowledge of the EHR system remains important. Without an understanding of the EHR system, its components, and how it works to facilitate continuity of care, it is difficult for a student to imagine innovative solutions to higher level problems in everyday practice. An understanding of the EHR system provides a foundation for a deeper understanding of healthcare information systems as the student gains more knowledge and experience. The present study examines students' learning after using the CDSS component of an EHR system that was implemented in our health informatics program to prepare the students for the healthcare workforce. In the process of using this CDSS component in the EHR system, students also accessed patient records to gain an understanding of clinical documentation in the EHR system.

\section{EXPERIENTIAL LEARNING IN TEACHING HEALTHCARE INFORMATION SYSTEMS}

Hence, in a health or medical informatics program, experiential learning with EHRs is essential. There are benefits to hands-on learning with EHRs. According to Jewer and Evermann (2015, p. 187), "advances in pedagogical approaches place emphasis on learning by doing." Jewer and Evermann elaborate and state that "passive learning, such as through lectures, has been shown to be inferior to experiential learning" (pp. 187-188) which is especially true for courses involving health IT. Jewer and Evermann (2015) have found experiential learning to be effective in teaching enterprise resource planning (ERP) systems and business process management. Monk and Lycett (2016) found that hands-on experience with ERP systems helped students understand business processes. Likewise, experiential learning with EHR systems can help students understand not only the EHR system and its functions but also healthcare processes. The latter is essential especially for students who do not have a healthcare background when they take health informatics courses. Also, conceptual knowledge gained through one information system is transferable (Jewer \& Evermann, 2015). Ayyagari (2011) and Jewer and Evermann (2015) point to evidence that conceptual knowledge gained from an information system is more important than the specific skills learned from a particular software package. Thus, the specific EHR system students use is less important than the conceptual knowledge gained which can be applied to any EHR system they encounter in the future.

Given the calls for EHR training in curricula and the significance of situational learning, we implemented an EHR system in our health informatics program to integrate practical experiences into our classes. Initially, we implemented the EHR system in our health informatics program to offer experiential learning to students (Ankem, Uppala, \& Dhawan, 2017). A description of the implementation of the EHR system can be found in Ankem, Uppala, and Dhawan (2017). In this paper, we are interested in finding if this integration is effective in delivering the expected experiential learning. Many of the descriptions of EHR integration in university education do not cover this evaluation aspect; that is, the effectiveness of EHR integration in curricula is rarely examined. In the few instances that it is examined, the evaluation does not appear to be empirical. For example, according to Borycki and colleagues, who implemented an elaborate model to integrate the EHR in education at the University of Victoria, hands-on experience with EHRs improved undergraduate health informatics students' competencies (Borycki et al., 2013). Within the same university, findings from an evaluation of a teaching approach indicated that the EHR can also be successfully integrated into 
problem-based medical education. However, it is not clear whether Borycki and colleagues conducted an empirical assessment of the effectiveness of EHR use in the education of students in health informatics as well as those in health professions.

Therefore, the plan in the present study is to employ an experimental method to investigate the effectiveness of EHR use in the instructional setting. Specifically, the CDSS component of the EHR system was chosen to deliver the handson experience and examine the effect on student learning. Students also accessed patient records to understand clinical documentation and healthcare processes. The experiment is described in detail in the methodology section and CDSS hands-on component that follows. The focus in the experiment is on one component of the EHR, the CDSS which generates patient specific assessments or recommendations to clinicians for consideration. Pre-test questions were administered to examine students' understanding of CDSS concepts and functionality. Then, students completed the CDSS hands-on component. The same questions were administered as a post-test, in addition to questions on student engagement, student learning, and student interest in learning more. An overview of the CDSS hands-on component that students completed follows the methodology section. The CDSS hands-on component includes medication ordering support and knowledge support tools of the CDSS. Data collected in February 2018 were tested for reliability and validity and analyzed using t-test, means, and standard deviations. The t-test was applied to find a difference in students' understanding of CDSS concepts and functionality between pre- and post-tests. Their open-ended responses to demonstrate understanding of CDSS concepts and functionality were assessed qualitatively to find if understanding increased from pre- to post-test. Means and standard deviations were used to examine student engagement, student learning, and student interest in learning more as a result of students' hands-on experience with the CDSS.

\section{METHODOLOGY}

Upon IRB approval of the study, students in two sections of an undergraduate health informatics course (total $=63$ ) were asked to complete a hands-on CDSS component for course credit in the first two weeks of February 2018. The course is required for nursing and allied health majors. The average age of participants in these courses is 23.9. The majority of participants are under the age of 25 while a small number are over the age of $25(15.3 \%$ are $25-34,7.7 \%$ are $35-49$, and $1.4 \%$ are 50 and over). Eighty-six percent of the participants were female and $14 \%$ were male. Many students already work in healthcare organizations and are familiar with healthcare operations to a certain extent but are learning about health IT. The questions asked were general, that is, not specific to a specific healthcare organization. There are students in these classes from other majors, including information systems, computer science, and communication. To complete the hands-on CDSS component, students worked in groups of 3. They were administered pre-test questions on their understanding of CDSS concepts and functionality in the first week (Table 1). Students listened to the lecture on EHRs which included a section on the CDSS and completed the assigned readings. At the end of the first week, they were asked to respond to the pre-test questions (Q1-Q5d).

In the second week, students were asked to complete the CDSS hands-on component. The CDSS hands-on component is described in the section that follows the methodology section. Toward the end of the second week (that is, after students completed the CDSS hands-on component), the same questions (Q1-Q5d) were administered as a post-test, along with additional questions to gauge whether the hands-on component was engaging and whether it contributed to their learning and generated an interest in learning more about the CDSS (Q6a-Q7c). Two students had dropped the course before completing the hands-on component, and five other students did not complete either the pre-test or the post-test. Hence, the sample included 56 participants. 
Table 1. Learning Outcome Measures

\begin{tabular}{|c|c|c|}
\hline \multirow[t]{2}{*}{$\begin{array}{l}\text { Understanding } \\
\text { Pre- and Post-Test }\end{array}$} & $\begin{array}{l}\text { Please discuss your understanding of the following: } \\
\text { - the clinical decision support system (CDSS) (Q 1) } \\
\text { - the place of the CDSS in a healthcare organization } \\
\text { (Q 2) } \\
\text { - how the CDSS relates to other components of the } \\
\text { EHR system (Q 3) } \\
\text { - how the CDSS can be useful to a healthcare } \\
\text { organization (Q 4) }\end{array}$ & Open-ended question \\
\hline & $\begin{array}{l}\text { Please rate the following: } \\
\text { - I have a good understanding of CDSS concepts. } \\
\text { (Q 5a) } \\
\text { - I am able to explain CDSS concepts to other } \\
\text { students. (Q 5b) } \\
\text { - I am able to use the CDSS. (Q 5c) } \\
\text { - I am able to make a case for using the CDSS to } \\
\text { others. (Q 5d) }\end{array}$ & $\begin{array}{l}\text { 7-point scale ranging from } \\
\text { "strongly disagree" to } \\
\text { "strongly agree" }\end{array}$ \\
\hline $\begin{array}{l}\text { Engagement } \\
\text { Post-Test }\end{array}$ & $\begin{array}{l}\text { Please rate the following: The class exercise to use } \\
\text { the CDSS ... } \\
-\ldots \text { Kept me absorbed (Q 6a) } \\
-\ldots \text { Held my attention (Q 6b) } \\
-\ldots \text { Excited my curiosity (Q 6c) } \\
-\ldots \text { Aroused my imagination (Q 6d) } \\
-\ldots \text { Was fun (Q 6e) } \\
-\ldots \text { Was interesting (Q 6f) } \\
-\ldots \text { Was engaging (Q 6g) } \\
-\ldots \text { Was useful (Q 6h) }\end{array}$ & $\begin{array}{l}\text { 7-point scale ranging from } \\
\text { "strongly disagree" to } \\
\text { "strongly agree" }\end{array}$ \\
\hline $\begin{array}{l}\text { Learning } \\
\text { Post-Test }\end{array}$ & $\begin{array}{l}\text { Please rate the following: The class exercise to use } \\
\text { the CDSS ... } \\
\text { - ... Increased my understanding of basic concepts } \\
\text { of the CDSS (Q 6i) } \\
-\ldots \text { Helped me to learn CDSS functions (Q } 6 \mathrm{j}) \\
-\ldots \text { Helped me to identify issues in the CDSS (Q } \\
\text { 6k) } \\
\text { - ... Helped me to interrelate important topics and } \\
\text { ideas in the CDSS (Q 61) } \\
\text { - ... Helped me to learn basic concepts of the } \\
\text { CDSS (Q 6m) }\end{array}$ & $\begin{array}{l}\text { 7-point scale ranging from } \\
\text { "strongly disagree" to } \\
\text { "strongly agree" }\end{array}$ \\
\hline $\begin{array}{l}\text { Learning Interest } \\
\text { Post-Test }\end{array}$ & $\begin{array}{l}\text { Please rate the following: } \\
\text { - I will discuss related topics outside the class (Q } \\
\text { 7a) } \\
\text { - I will do additional reading on related topics (Q } \\
7 \text { b) } \\
\text { - I will do some thinking for myself about related } \\
\text { issues (Q 7c) }\end{array}$ & $\begin{array}{l}\text { 7-point scale ranging from } \\
\text { "strongly disagree" to } \\
\text { "strongly agree" }\end{array}$ \\
\hline
\end{tabular}

Note. Adapted from "Enhancing Learning Outcomes through Experiential Learning: Using Open-Source Systems to Teach Enterprise Systems and Business Process Management" by J. Jewer and J. Evermann, 2015, Journal of Information Systems Education, 26(3), p. 193. Copyright (C2015 by the Education Special Interest Group (EDSIG) of the Association of Information Technology Professionals. 


\section{Issues in Information Systems \\ Volume 20, Issue 3, pp. 194-207, 2019}

Table 1 presents the questions included in each category: understanding of CDSS concepts and functionality (Q1Q5d), engagement experienced while working on the CDSS hands-on component (Q6a-Q6h), learning that occurred as a result of doing the CDSS hands-on component (Q6i-Q6m), and interest in learning more about the CDSS after using the EHR system to do the CDSS hands-on component (Q7a-Q7c). The following hypotheses state the expectations regarding student outcomes upon students using the EHR system to do the CDSS hands-on component in this research.

$\mathrm{H}_{1}$ : Students' understanding of CDSS concepts and functions increased from pre-test to post-test.

$\mathrm{H}_{2}$ : Student engagement increased at post-test.

$\mathrm{H}_{3}$ : Student learning increased at post-test.

$\mathrm{H}_{4}$ : Students' interest in learning more about the CDSS increased at post-test.

\section{CDSS HANDS-ON COMPONENT}

Students reviewed the EHR of a patient suspected of dengue fever. The simulated patient scenario of the EHR is presented in the appendix (Table A1). Based on a case published in an EHR training manual (Gartee, 2017), the patient scenario was further developed through collaboration with a physician from the local hospital system. To start, students were asked to identify and describe clinical documentation in the EHR. Then, students were asked to complete two tasks using the CDSS.

In Task 1, students changed the physician's recommendation of over-the-counter Monistat (miconazole) to a prescription of Diflucan (fluconazole) at a one-time dose of $150 \mathrm{mgs}$ to be taken if symptoms of early yeast vaginitis increase in the patient. As the patient has visited primarily for high fever, severe headache, and muscle/joint pain, early yeast vaginitis is not one of the chief complaints (Table A1). The patient, however, is concerned about early yeast vaginitis. When students change the order, they see a CDSS alert for a major interaction with Citalopram that the patient is taking for anxiety. Upon reviewing the CDSS alert, students proceed to retain the original recommendation of over-the-counter Monistat (miconazole) for the patient (Figure 1).

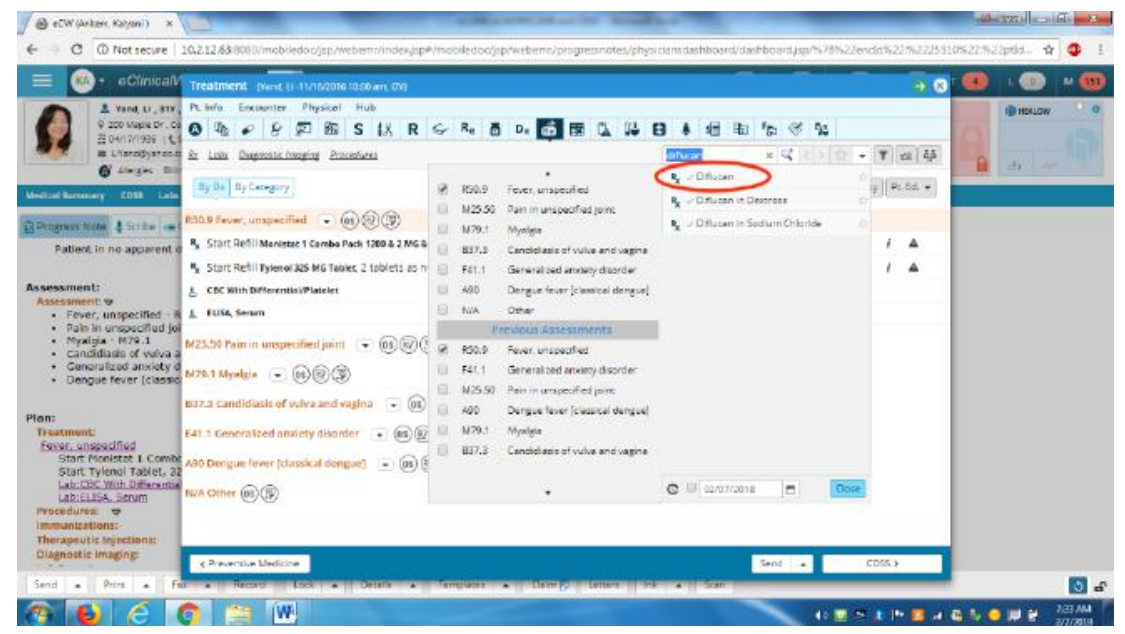

Figure 1. eClinicalWorks Order Entry

In Task 2, students investigated the reason for the physician's recommendation to the patient to avoid aspirin products, including Excedrin. If a knowledge support resource had been integrated in the EHR system, students could readily consult this resource to determine the reasoning for the recommendation. A knowledge support resource such as iCONSULT is offered as part of the Epic EHR system which is widely considered the industry leader: "iCONSULT 


\section{Issues in Information Systems}

Volume 20, Issue 3, pp. 194-207, 2019

delivers real-time clinical decision support by putting evidence-based, point-of-care clinical reference content directly into the electronic workflow of health practitioners" (Elsevier, 2006). We implemented eClinicalWorks, an EHR system which allows the integration of UpToDate, a different knowledge support resource; however, the university does not subscribe to UpToDate for such integration to be undertaken. The preceding was explained to students.

Hence, students were asked to access the Centers for Disease Control and Prevention (CDC) site in the browser and review clinical decision support material on dengue fever to explain the reasoning for the recommendation to avoid aspirin. The idea is for students to access a knowledge support resource and understand that dengue fever increases the risk of bleeding which is exacerbated by Excedrin (analgesic containing aspirin); therefore, the physician's recommendation is to avoid Excedrin and other aspirin products.

\section{RESULTS}

Testing students' understanding of CDSS concepts and functionality included qualitative questions (Q1-Q4) and quantitative questions (Q5a-Q5d). The qualitative questions inquired about their understanding of the CDSS, how the CDSS fits in a healthcare organization, how it relates to other components of the EHR system, and its usefulness in a healthcare organization (Table 1). Only 46 responses to Q1-Q4 were useable due to missing or incomplete discussions by some students when responding to these questions. Students' discussions of CDSS concepts, functionality of the CDSS as a component of the EHR, and place and usefulness of the CDSS in a healthcare organization were compared between pre- and post-tests. The improvement in their responses to each of these questions from pre-test to post-test was scored on a scale of $0=$ no improvement, $1=$ some improvement, and $2=$ significant improvement. To test interrater reliability, two of the three authors scored the responses independently. Kappa coefficient of 0.44 showed moderate agreement. After an in-depth discussion of the ratings, the researchers arrived at an increased Kappa coefficient 0.72 . Table 2 shows the mean improvement score for each open-ended response (Q1-Q4). T-tests point to significant improvement $(\mathrm{p}<.01)$ in students' understanding of CDSS concepts, functionality of the CDSS, and place and usefulness of the CDSS in a healthcare organization.

Table 2. Understanding of the CDSS

\begin{tabular}{|l|c|c|c|}
\hline Discuss your understanding of... & $\mathbf{N}$ & Mean & Std. Dev. \\
\hline $\begin{array}{l}\text { (Q1) the clinical decision support } \\
\text { system (CDSS) }\end{array}$ & 46 & 1.17 & .85 \\
\hline $\begin{array}{l}\text { (Q2) the place of the CDSS in a } \\
\text { healthcare organization }\end{array}$ & 46 & 1.11 & .92 \\
\hline $\begin{array}{l}\text { (Q3) how the CDSS relates to other } \\
\text { components of the EHR system }\end{array}$ & 46 & 1.15 & .92 \\
\hline $\begin{array}{l}\text { (Q4) how the CDSS can be useful to } \\
\text { a healthcare organization }\end{array}$ & 46 & 1.48 & .81 \\
\hline
\end{tabular}

Questions (Q5a-Q5d) used a 7-point Likert scale to measure students' understanding of CDSS concepts, ability to explain CDSS concepts, ability to use the CDSS, and ability to convince others to use the CDSS. A principal component analysis of the data identifies one factor at pre-test as well as at post-test (Table 3). A t-test shows that their understanding of CDSS concepts and functionality improved significantly at post-test $(p<.01)$. It is significantly higher at post-test. Figure 2 illustrates boxplots of students' understanding of CDSS concepts and functionality at preand post-test. To reiterate, these questions probed students' understanding of CDSS concepts, ability to explain CDSS concepts, ability to use the CDSS, and ability to convince others to use the CDSS. 


\section{Issues in Information Systems}

Volume 20, Issue 3, pp. 194-207, 2019

Table 3. Principal component analysis - students' understanding of CDSS concepts and functionality at pre- and post-test

\begin{tabular}{|l|c|c|}
\hline & $\begin{array}{c}\text { Pre-test } \\
\text { Factor 1 }\end{array}$ & $\begin{array}{c}\text { Post-test } \\
\text { Factor 1 }\end{array}$ \\
\hline Q5a & .868 & .899 \\
\hline Q5b & .820 & .931 \\
\hline Q5c & .664 & .670 \\
\hline Q5d & .801 & .863 \\
\hline
\end{tabular}

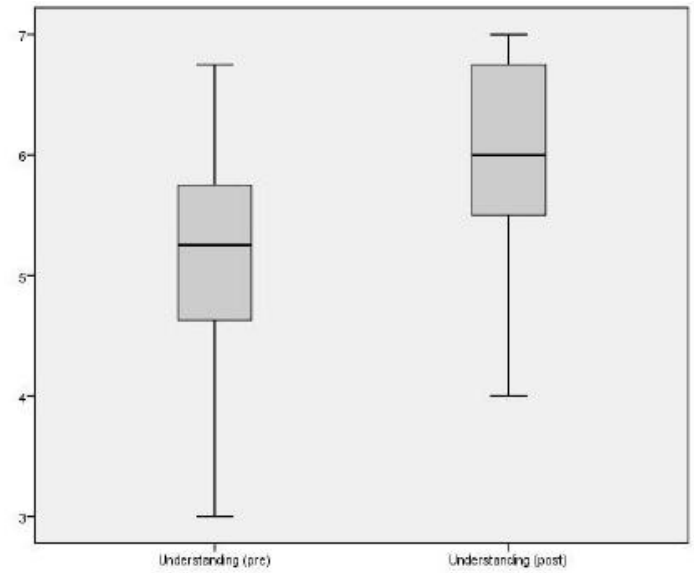

Figure 2. Boxplots - students' understanding of CDSS concepts and functionality at pre- and post-test

Questions Q6a-Q6m were designed to capture students' perception of engagement and learning at post-test upon using the EHR system to do the hands-on CDSS component. A principal component analysis of the responses to these questions identifies two components (Table 4). These questions were measured on a 7-point Likert scale. A maximum likelihood estimation of the same data confirms the two components. Q6h was separated from this analysis as it asked about usefulness and was considered to deviate conceptually when compared to the other items in Q6a through Q6m. These other items asked about whether the hands-on component held their attention; aroused curiosity or imagination; was engaging, interesting, or fun; increased understanding of CDSS concepts; helped in learning CDSS functions and identifying issues in the CDSS; and helped to interrelate CDSS topics and concepts. In Table 4 below, the factor loadings are displayed for the two factors which are referred to here as engagement and learning.

Table 4. Principal component analysis - student engagement and student learning at post-test

\begin{tabular}{|l|c|c|}
\hline & Factor 1 & Factor 2 \\
\hline Q6a & .740 & .420 \\
\hline Q6b & .847 & .322 \\
\hline Q6c & .754 & .467 \\
\hline Q6d & .797 & .415 \\
\hline Q6e & .844 & .351 \\
\hline Q6f & .866 & .328 \\
\hline Q6g & .763 & .439 \\
\hline Q6i & .494 & .784 \\
\hline Q6j & .434 & .831 \\
\hline Q6k & .268 & .837 \\
\hline Q61 & .392 & .837 \\
\hline Q6m & .422 & .806 \\
\hline
\end{tabular}




\section{Issues in Information Systems}

Volume 20, Issue 3, pp. 194-207, 2019

Figure 3 illustrates the boxplots of engagement (Q6a-Q6g), usefulness (Q6h), and learning (Q6i-Q6m). A t-test shows that the engagement students experienced with the hands-on component is significantly higher than the midpoint of the scale $(\mathrm{p}<.01)$. Also, a t-test shows that the hands-on component significantly increased students' learning of CDSS concepts, CDSS functionality and issues, and the interrelationships between CDSS topics and ideas $(\mathrm{p}<.01)$. Students' perceived usefulness of the hands-on component is also significantly higher than the midpoint of the scale $(\mathrm{p}<.01)$.

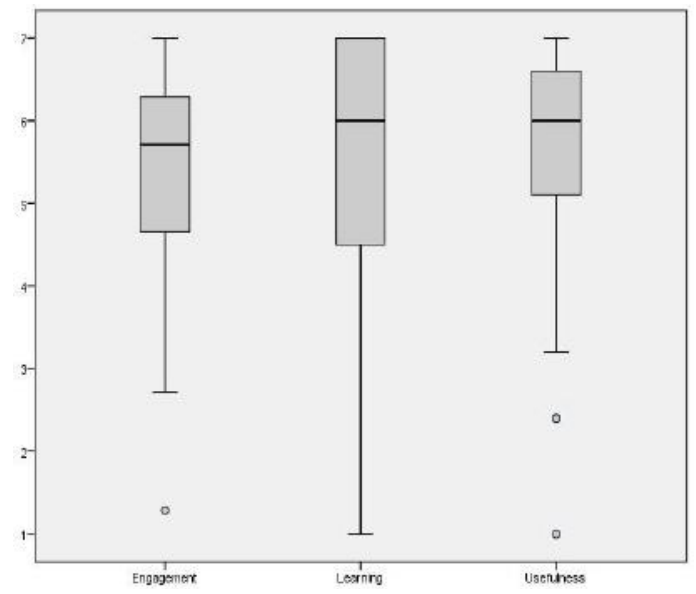

Figure 3. Boxplots - students' perception of engagement, learning and usefulness at post-test

The last three questions (Q7a-Q7c) in Table 1 captured students' interest in learning more about the CDSS and were measured on a 7-point Likert scale. The t-tests revealed that while Q7a-Q7b are similar, Q7c is different $(\mathrm{p}<.01)$. Q7a-Q7b asked about students' willingness to discuss related topics outside of the class and to do additional reading (beyond that assigned in the class) while Q7c asked about whether they will think further about related topics. Figure 4 displays boxplots of the responses to each of these three questions on student interest in extending their learning. Students' interest in learning more about CDSS related topics was consistently higher than the midpoint of the scale.

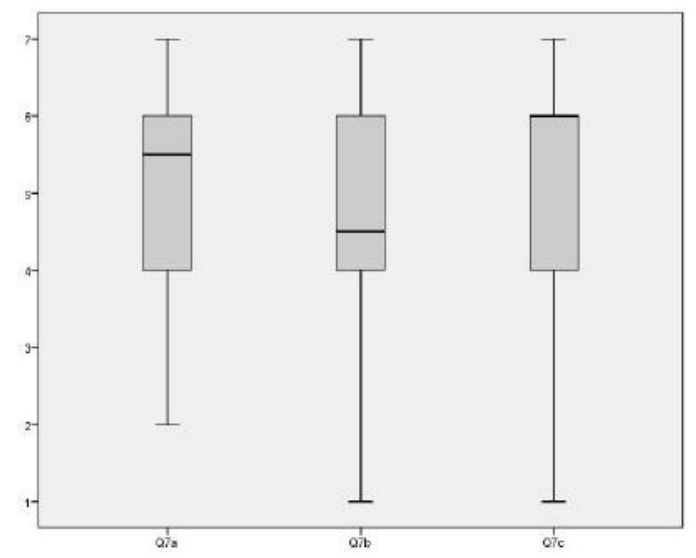

Figure 4. Boxplots - students' interest in extending learning at post-test

\section{DISCUSSION}

The findings in the present study show that experiential learning significantly contributes to students' understanding of the CDSS in the EHR system. In prior research, Jewer and Evermann (2015) illustrated similar results in learning about ERP systems. Monk and Lycett (2016) found that experiential learning through ERP systems is effective in teaching business processes. The findings in the present study reinforce the benefits students gain in understanding 


\section{Issues in Information Systems \\ Volume 20, Issue 3, pp. 194-207, 2019}

health IT and healthcare processes when given the opportunity for experiential learning. Students' understanding of CDSS concepts and functionality significantly improved after they used the EHR system to work on the CDSS handon component. The preceding was substantiated in structured as well as open-ended responses where students discussed their understanding of the CDSS as a component of the EHR and its usefulness to the healthcare organization. Students found the CDSS hands-on component to be engaging. Students also felt the CDSS hands-on component contributed to their learning and generated an interest in exploring CDSS topics further.

Students in health informatics programs, especially in those programs that are housed in information systems departments, often gain the hands-on component with healthcare information systems though internships and practicums. While such an arrangement may offer the opportunity to gain the essential experience, not all students are exposed to similar healthcare settings and to similar health IT experiences when engaging in a practicum or internship. Typically, it is infeasible to place all of the students in a healthcare organization where the students can observe and learn the application of healthcare IT in a substantive manner. Hence, it is imperative for health informatics programs, especially those housed in information systems departments, to prepare students in a structured, systematic way that is consistent in not only teaching healthcare information systems but also applying pedagogy that is experiential. Also, it is important to realize that an understanding of the EHR is necessary for students before they can delve into more complexities that will allow them to tackle the deeper problems in everyday healthcare practice.

Literature has pointed that hands-on learning is superior to the lecture mode. Students who are taught only through lectures are incapable of perceiving or experiencing the full understanding of healthcare information systems or the underlying concepts. Teaching complex healthcare information systems skills through experiential learning as undertaken at the School of Health Information Science at the University of Victoria is an elaborate undertaking. It involves elaborate planning and preparation to set up the complex infrastructure required to integrate the EHR in teaching nursing, medical, and health informatics students (Borycki et al., 2009; Borycki et al., 2011; Borycki et al., 2013; Kushniruk et al., 2012; Kushniruk et al., 2014). Such an undertaking requires substantial resources in the way of faculty expertise, time, and funding. With dedication and commitment to experiential learning, this planning and set-up are possible; however, it may be necessary to install and experiment with an EHR system prior to delving into a more complex healthcare IT infrastructure.

Drawing upon the results, we recommend, routine integration of health IT in health informatics curricula. Also, as information technologies continue to develop in medicine and in nursing, healthcare IT integration that becomes routine in healthcare curricula to prepare future healthcare professionals is essential to leverage the capabilities and functionalities of the EHR system and to realize improved patient outcomes. Especially in health informatics, a discipline focused on the development and application of health IT, the education of professionals must be predicated on experiential learning.

\section{CONCLUSION}

The participants' opinions and their knowledge in pre- and post-responses indicate that health IT integration is effective in health informatics curricula. The preparation of health informatics professionals rooted in robust experiential learning will enable them to not only start their practice with the requisite knowledge but also contribute at a higher level in reaching health IT solutions in delivering and managing healthcare, such as the facilitation of ideal approaches in leveraging healthcare data analytics to improve patient outcomes. It is important to not only integrate health IT but also evaluate the outcomes of such integration in curricula. The present research offers a model and accomplishes the empirical evaluation to inform educators of the effectiveness of experiential learning in teaching health informatics. An evaluation reaffirms the value of health IT in curricula and encourages others to follow suit. It also encourages educators to make necessary changes to the curricula based on student feedback.

\section{ACKNOWLEDGEMENTS}

The authors wish to thank Dr. Patrick Kunkler, MD, at St. Elizabeth Healthcare for generously sharing his expertise and valuable time in writing the patient scenario used in creating the CDSS hands-on component. 


\section{Issues in Information Systems}

Volume 20, Issue 3, pp. 194-207, 2019

\section{REFERENCES}

Ankem, K., Uppala, V., \& Dhawan, A. (2017). Electronic health record system implementation in a health informatics program: A case study. Journal of the Midwest Association for Information Systems, 2.

AHRQ. (2018). Clinical decision support. Retrieved January 20, 2019 from https://www.ahrq.gov/professionals/prevention-chronic-care/decision/clinical/index.html

Ayyagari, R. (2011). Hands-on ERP learning: Using OpenERP, an alternative to SAP. Journal of Information Systems Education, 22(2), 122-123.

Biagioli, F. E., Elliot, D. L., Palmer, R. T., Graichen, C. C., Rdesinski, R. E., Ashok Kumar, K., ... \& Tysinger, J. W. (2017). The electronic health record objective structured clinical examination: Assessing student competency in patient interactions while using the electronic health record. Academic Medicine, 92(1), 8791.

Borycki, E. M., Kushniruk, A. W., Joe, R., Armstrong, B., Otto, T., Ho, K., \& Frisch, N. (2009). The University of Victoria interdisciplinary electronic health record educational portal. Studies in Health Technology and Informatics, 143, 49-54.

Borycki, E. M., Joe, R. S., Armstrong, B., Bellwood, P., \& Campbell, R. (2011). Educating health professionals about the electronic health record (EHR): Removing the barriers to adoption. Knowledge Management and E-Learning: An International Journal, 3(1), 51-62.

Borycki, E. M., Griffith, J., Reid, P., Kushniruk, A. W., \& Kuo, M. -H. (2013). Do electronic health records help undergraduate health informatics students to develop health informatics competencies? Studies in Health Technology and Informatics, 192, 1106.

Elsevier. (2006, January 20). Elsevier Announces iCONSULT to be Offered as Part of Epic Electronic Health Record [Press Release]. Retrieved December 10, 2017 from https://www.elsevier.com/about/pressreleases/clinical-solutions/elsevier-announces-iconsult-to-be-offered-as-part-of-epic-electronic-healthrecord

Gartee, R. (2017). Electronic health records: Understanding and using computerized medical records. London: Pearson Education.

HealthIT.gov. (2018a). Clinical decision support. Retrieved January 20, 2019 from https://www.healthit.gov/topic/safety/clinical-decision-support

HealthIT.gov. (2018b). What is an Electronic Health Record (EHR)? Retrieved March 20, 2018 from https://www.healthit.gov/faq/what-electronic-health-record-ehr

Herbert, V. M., \& Connors, H. (2016). Integrating an academic electronic health record: Challenges and success strategies. CIN: Computers, Informatics, Nursing, 34(8), 345-354.

HIMSS. (2017). Electronic Health Records. Retrieved December 15, 2017 from http://www.himss.org/library/ehr

Jewer, J., \& Evermann, J. (2015). Enhancing learning outcomes through experiential learning: Using open-source systems to teach enterprise systems and business process management. Journal of Information Systems Education, 26(3), 187-201. 


\section{Issues in Information Systems}

Volume 20, Issue 3, pp. 194-207, 2019

Kushniruk, A., Borycki, A., Joe, R., Otto, T., Armstrong, B., \& Ho, K. (2012). Integrating electronic health records into medical education: Considerations, challenges, and future directions. In K. Ho, S. Jarvis-Selinger, H. Novak Lauscher, J. Cordeiro, \& R. Scott (Eds.), Healthcare Delivery in the Information Age. Technology enabled knowledge translation for eHealth (pp. 21-31). New York: Springer.

Kushniruk, A. W., Kuo, M. -H., Parapini, E., \& Borycki, E. M. (2014). A virtual platform for electronic health record (EHR) education for nursing students: Moving from in-house solutions to the cloud. Studies in Health Technology and Informatics, 201, 48-54.

Milano, C. E., Hardman, J. A., Plesiu, A., Rdesinski, R. E., \& Biagioli, F. E. (2014). Simulated electronic health record (Sim-EHR) curriculum: Teaching EHR skills and use of the EHR for disease management and prevention. Academic Medicine, 89(3), 399-403.

Monk, E. F., \& Lycett, M. (2016). Measuring business process learning with enterprise resource planning systems to improve the value of education. Education and Information Technologies, 21(4), 747-768.

Otto, A., \& Kushniruk, A. (2009). Incorporation of medical informatics and information technology as a core component of undergraduate medical education - time for change! Studies in Health Technology and Informatics, 143, 62-67.

Welcher, C. M., Hersh, W., Takesue, B., Stagg Elliott, V., \& Hawkins, R. E. (2018). Barriers to medical students' electronic health record access can impede their preparedness for practice. Academic Medicine, 93(1), 4853.

Wilson, E. V., \& Tulu, B. (2010). The rise of a health-IT academic focus. Communications of the ACM, 53(5), 147150.

Zhang, C., Reichgelt, H., Rutherfoord, R. H., \& Wang, A. J. A. (2014). Developing health information technology (HIT) programs and HIT curriculum: The Southern Polytechnic State University experience. Journal of Information Systems Education, 25(4), 295-303. 


\section{APPENDICES}

Table A1. Patient Scenario

CC: High fever, severe headache, and muscle/joint pain

Li Yand is a 31-year-old female who has developed a high fever, a severe headache mostly behind eyes (retrobulbar), and more muscle than joint pain after a relaxing vacation in Mexico. She believes she contacted the flu from the close confines of the plane during the flight home. She was in Cancun, Mexico for 6 days and returned home 5 days ago. She has had a high fever for the last 2 days along with a severe retrobulbar headache, and myalgia (muscle aches). Extra Strength Excedrin (acetaminophen-aspirin-caffeine) is not working to relieve these symptoms. No sinus pain, swollen glands, earache, nasal discharge, sore throat, cough, or other signs of cold. No chest pain or dyspnea. No present nausea, vomiting, diarrhea or constipation. No known exposure to anyone with similar symptoms including her boyfriend who was also with her this entire trip. She did not use insect repellant and sustained multiple mosquito bites during her vacation. No other rash noted.

Due to the warm environment and wet clothing on vacation, she believes she may be starting to get a vaginal yeast infection. She has some mild vaginal itching with no discharge. She denies any history of sexually transmitted disease and has been in a monogamous relationship for over 2 years. She wants to have a one-time dose of Diflucan to use if vaginal yeast symptoms become more prevalent - she has used it in the past with no problems.

She is on birth control pills (BCP) and denies any chance of pregnancy. She has been on Citalopram for general anxiety disorder for the last 3 years with no problems.

Review of systems: no respiratory, cardiac, or gastrointestinal (GI) symptoms

Surgical history: tonsillectomy at age 7 years and appendectomy at age 14 years

Meds - BCP, Citalopram, Extra Strength Excedrin

All: none $=$ NKDA

Social - lives with her boyfriend of 3 years. No tob. Occasional wine - a glass of wine per week - negative CAGE. Works as accountant.

Vitals: Weight: 110 pounds. Height: $5 \mathrm{ft}$. 2inches. BMI $-20.1 \mathrm{BP}=140 / 90$, Pulse is 96 and regular. Resp : 24 per minute, Temp $=104.0$

Physical exam - Patient in no apparent distress but appears ill. Patient is alert and cooperative.

HEENT: Ears: TM normal bilaterally. Sclera is non-icteric and there is no erythema of the conjunctiva. Sinuses are non-tender. Nasal mucosa with no erythema or drainage. Mouth with mucous membrane pink and moist with no swelling or exudate. Neck is supple with no LN, carotid bruits, or thyroid abnormalities. Full ROM of neck with no meningeal signs

Lungs are clear with OK air movement. No rales, rhonchi, or wheezes

$\mathrm{CV}$ with regular rate and rhythm - no murmurs, rubs, or gallops

Abdomen is non-tender with no mass or HSM, no hernia - well healed laparoscopic scars.

Extremities - no edema, Ok pulses, Ok ROM of joints of upper and lower extremities with no swelling, warmth, or erythema

Skin - no jaundice (icterus). Multiple diffuse, resolving erythematous blisters are consistent with mosquito bites. No present rash.

Labs - serum ELISA and/or RT-PCR for Dengue Fever, CBC with differential pending

Assessment: Dengue Fever A00-B99 / febrile illness - R50.9 / arthralgia - M25.50 / myalgia - M79.1 / yeast vaginitis - B37.3 / generalized anxiety disorder (GAD) - F41.1 
Treatment: Drink plenty of fluids - stay well-hydrated. Stop the Excedrin and avoid all products containing aspirin and nonsteroidal anti-inflammatory drugs (NSAIDs). Use Tylenol (acetaminophen) and tepid sponge baths.

She is to call with status in 2 days for blood test results - sooner if symptoms increase or new symptoms present. Discussed usual resolution of this disease with risk of progression to severe dengue that would mostly likely occur in the next 5 to 7 days which includes persistent vomiting, severe abdominal pain, difficulty breathing, a change in mental status, a decrease in blood pressure, and a decrease in platelet count on CBC.

Use protection to avoid mosquito bites while febrile and to avoid mosquito-transmitted diseases in future - use insect repellent with at least $20 \%$ DEET regularly and wear clothes to cover trunk, arms, and legs especially during early morning and later afternoon.

[Concerning possible early yeast vaginitis - tried to prescribe Diflucan (fluconazole) $150 \mathrm{mg}$ one time dose to use if symptoms increased - Alert occurred which warned of possible major interaction with Citalopram - thus, recommended patient use over-the-counter Monistat (miconazole) vaginal cream as directed, which does not appear to have this potential interaction]

Problem list - GAD - Ok on Citalopram

Preventive Medicine - to have regular check-ups 